\title{
A WEB-BASED PLATFORM FOR REMOTE SENSING IMAGE ANNOTATION
}

\author{
A. Garcia-Pedrero ${ }^{\mathrm{a}, \mathrm{b}, *}$, C. Gonzalo-Martín ${ }^{\mathrm{a}, \mathrm{b}}$, M. Lillo-Saavedra ${ }^{\mathrm{c}, \mathrm{d}}$, C. Ortiz-Toro ${ }^{\mathrm{a}, \mathrm{b}}$ \\ ${ }^{a}$ Center for Biomedical Technology, Universidad Politécnica de Madrid, Campus de Montegancedo, \\ Pozuelo de Alarcón 28233, Spain - \{am.garcia\}@alumnos.upm.es. \\ ${ }^{\mathrm{b}}$ Escuela Técnica Superior de Ingenieros Informáticos, Universidad Politécnica de Madrid, Campus de Montegancedo, \\ Boadilla del Monte 28660, Spain \\ ${ }^{\mathrm{c}}$ Faculty of Agricultural Engineering, University of Concepción, Chile \\ ${ }^{\mathrm{d}}$ Water Research Center for Agriculture and Mining, CRHIAM, University of Concepción, Chile
}

KEY WORDS: Image annotation, object-based, active learning

\begin{abstract}
:
The generation of labeled data for training automated methods used in the analysis of remote sensing images is a challenging task. Approaches as Active Learning aim to perform accurate classifications in a scenario of a few annotated data. These approaches generally require the interaction between the user and the machine learning method during training phase. However, in the remote sensing area, it is difficult to find a tool that facilitates this interaction. In this work, an interactive web-based platform to perform the training of method for remote sensing image annotation by means of an active learning approach is proposed. The platform integrates open-source GIS technologies and object-based approach in order to facilitate the interaction between the user and the active learning approach. While some usability aspects should be improved, the obtained results show the potential of the proposed platform.
\end{abstract}

\section{INTRODUCTION}

The daily generation of terabytes of Earth-Observation data opens an opportunity to both industry and scientific communities to fully exploit these data in order to provide services to end-users (Ma et al. 2015). However, the analysis of these voluminous data is not possible by manual means, and therefore it is required the use of automatic and semiautomatic tools. In this regard, several machine learning and pattern recognition techniques have been used in remote sensing (RS) applications in order to facilitate the image annotation. During training step, these methods mostly require a large amount of annotated (labeled) samples that provides a good statistical separability of the classes to provide final accurate results (Xia et al. 2015).

Nevertheless, the generation of that labeled (annotated) collection is a challenging task, especially for large area mapping (Olofsson et al. 2014). In this context, Active Learning (AL) approaches aim to perform accurate classifications in a scenario of a few annotated data (Stumpf et al. 2014, Tuia and Munoz-Mari 2013 Crawford et al. 2013, Tuia et al., 2009). In a basic AL scheme, an user, also called oracle, provides annotated information and knowledge about the desired classes, while the classifier provides both its own interpretation of the distribution of the classes and the most relevant observations that are needed in order to solve the discrepancies encountered during training phase (Crawford et al. 2013). As one can imagine, the interaction between the user and the machine learning algorithm during training phase is fundamental.

To the best of our knowledge, there is not a platform that facilitates this interaction for remote sensing image annotation. In this work, we propose an interactive web-based platform to perform AL approach for remote sensing image annotation. The platform integrates open-source GIS technologies to display raster and vector datasets in a web browser, while internally a machine learning method is trained through AL approach. The proposed platform uses image-objects as minimum processing units instead of pixels.

${ }^{*}$ Corresponding author
Image-objects are labeled during AL process according to a set of classes established a-priori by the user. This reduces dramatically the number of instances to process in very-high resolution remote sensing images.

The rest of the paper is organized as follows. Section 2 describes the architecture of the system. In Section 3 the methodology adopted for the development of the proposed platform is described. Section 4 shows the results of a small experiment to test the proposed platform. Finally, main conclusions are given in Section 5

\section{SYSTEM ARCHITECTURE}

The proposed architecture (Figure 1 follows a web-based application scheme consisting of two main layers (server and client layers) and several modules. Each module has a different purpose, and is implemented using technology based on open standards and free software.

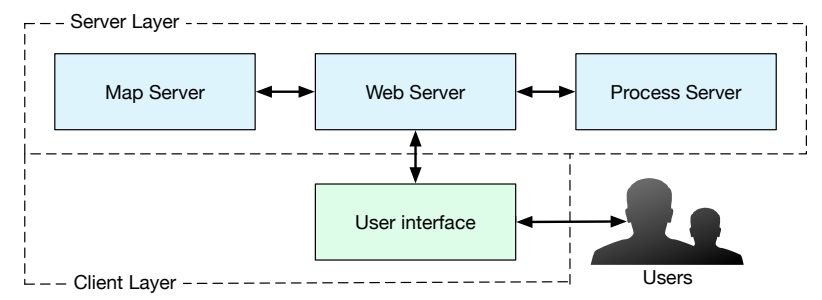

Figure 1. Architecture of the proposed platform.

\subsection{Server Layer}

This layer represents the main component in the system. It consists of three sub-modules: Map Server, Web Server, and Process Server modules.

The Map Server module contains the source imagery (raster) and vector data to be used by the system. A spatial database was implemented to optimize storage and query vector data. PostgreSQL 1

1 https://www. postgresql.org 
with PostGIS ${ }^{2}$ plugin was selected as management system of this database. GeoServer ${ }^{3}$ was chosen for rendering raster and vector data. It can handle data directly from most common spatial databases including PostgreSQL/PostGIS databases, as well as different raster formats such as GeoTIFF.

The Web Server module hosts the source code of the application developed using HTML5, Django, Python, JavaScript and CSS programming languages. It deals with incoming traffic and requests from client browsers. For this purpose, Nginx ${ }^{4}$ web server is used, which allows to serve static content quickly and efficiently, has a low memory usage (ideal for virtual environments), and offers a free-of-charge license. Moreover, Python scripts are used along the server layer for managing the communications between the users and the Web Server module, and between the last and the Process Server module for processing the data (e.g., segmentation, classification). Django is used to provide easy configuration management platform and connect to the database.

The Process Server module is mainly used for processing tasks such as image segmentation, and classification. This module receives the processing requests from Web Server module, which in turn receives from users.

In this work, all these modules are housed in the same computer, therefore they are limited by the capabilities of this equipment. For developing and testing this will not cause any problem, however for production applications that usually requires more capacity, this modules can be easily distributed in different machines using technologies such as Docker ${ }^{5}$

\subsection{Client Layer}

This layer defines the interactions between the users through an internet browser and the proposed system. It consists of a User Interface module, which is a web page implemented using AJAX and Javascript technologies. They are basically codes running on the user's computer, in particular in the web browser, which facilitate user interaction with the platform. In this regard, Openlayers $3^{6}$ an open-source Javascript library, was used to create interactive maps in the web browser with no server-side dependencies. It provides a complete control of map controls, map events, map layers, among others features. These features were exploited to display geospatial data (vector and raster) as a map.

Other libraries used to facilitate the creation of the web page were Jquery 7 , and Bootstran ${ }^{8}$ Jquery eased the implementation of manipulation operations and AJAX requests, while Bootstrap provided the framework to design a responsive front-end.

\section{METHODOLOGY}

As mentioned in Section 1 , the proposed platform aims to facilitate the process of active learning through an interactive process between the user and the classification method. Moreover, to ease and reduce the time of sampling, an object-based approach was implemented. Thus, the user instead of selecting pixels by manually delineating objects, simply selects the previously generated segments. As can be shown in Figure 2 in addition to the user interface (web page), two other main submodules were implemented: image segmentation and active learning. These submodules are explained in detail below.

\footnotetext{
2 http://postgis.net

3 http://geoserver.org

4 https://www.nginx.com

5 https://www.docker.com

6 http://openlayers.org

7 http://jquery.com

$\varepsilon$ http://getbootstrap.com
}

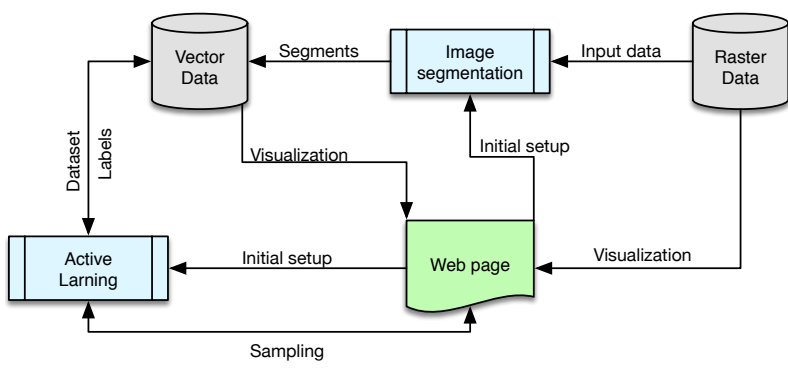

Figure 2. Flowchart of the methodology adopted to implement the platform.

\subsection{Image segmentation}

A superpixel method was selected to segment multispectral imagery. These type of methods produces a controlled over-segmentation of the image; in this regard, the generated superpixels tend to be similar in size and color, as well as belonging to only one object. In this work, a modified version of the SLIC algorithm (Achanta et al. 2012) for segmenting multispectral images (Garcia-Pedrero et al. 2015) was implemented. This method has two configuration parameters: a compactness factor and the superpixel size. The first one balances spectral proximity and space proximity, while the second one, as its name suggests, controls the number of pixels that in average comprises each superpixel.

\subsection{Active Learning}

The user-classifier interaction of AL approach is described as follows: 1) The user provides a small dataset of labeled regions. 2) The classifier learns the class distribution of the labeled data. 3) Random non-labeled regions are submitted to the classifier, which determines the regions with maximum uncertainty that should be labeled by the user. The last process is known as query. 4) Steps 1 to 3 are repeated until the classifier is trained. 5) Finally, the classifier is used to annotate the entire image.

Label Propagation (LP) algorithm (Bengio et al. 2006), implemented in Pedregosa et al. (2011), was selected as the classification method to be trained through an AL approach. LP is a graph-based method which main assumption is that instances occupying the same manifold are very likely to share the same label. Thus this method propagates labels of the labeled data to the unlabeled data instances according to the intrinsic data manifold structures found in the training data. There is currently no feature extraction module. Therefore, the segments are only characterized using the mean value of the pixels that they contain.

\subsection{User Interface}

The user interface, shown in Figure 3 incorporates the following features: (1) It allows the selection of different color composites for visualization. (2) It allows to locate a particular point of the image by geographical coordinates. (3) Tracking location is available. (4) It allows to show and hide the object (segment) boundaries. (5) It allows to label one or more segments at same time (Figure 4 . This operation is only possible if the object boundaries are visible. (6) It allows zoom in and zoom out operations for better viewing.

A configuration screen is also available to set the parameters necessary for the proper functioning of the platform. These parameters include: (1) the different color composites; (2) the parameters of the segmentation and classification algorithms; (3) AL parameters which include the number of labeled objects required to start the process, the number of elements (regions with higher uncertainty) to be manually labeled during a AL request (query), and the number of requests (iterations) necessary to complete the training. 


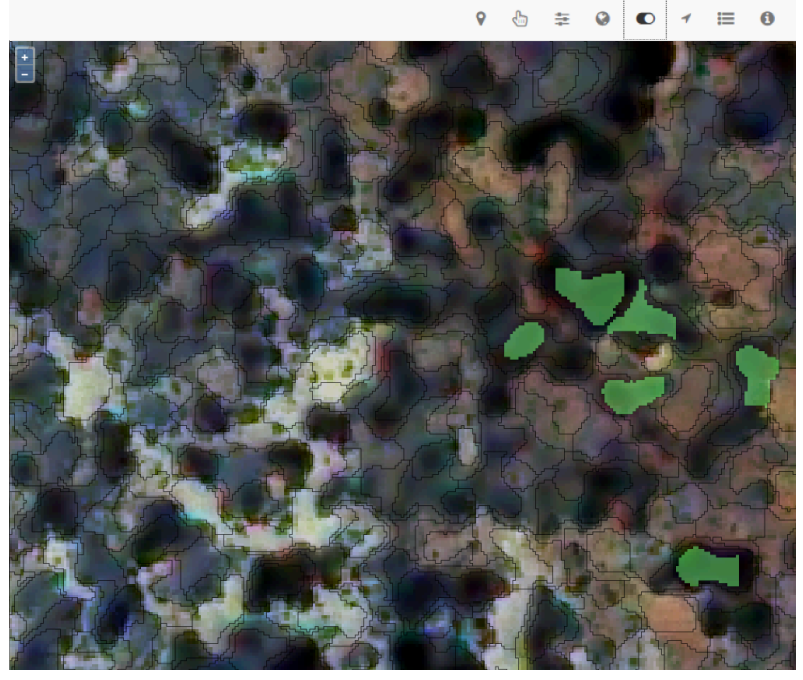

Figure 3. Overview of the proposed platform.

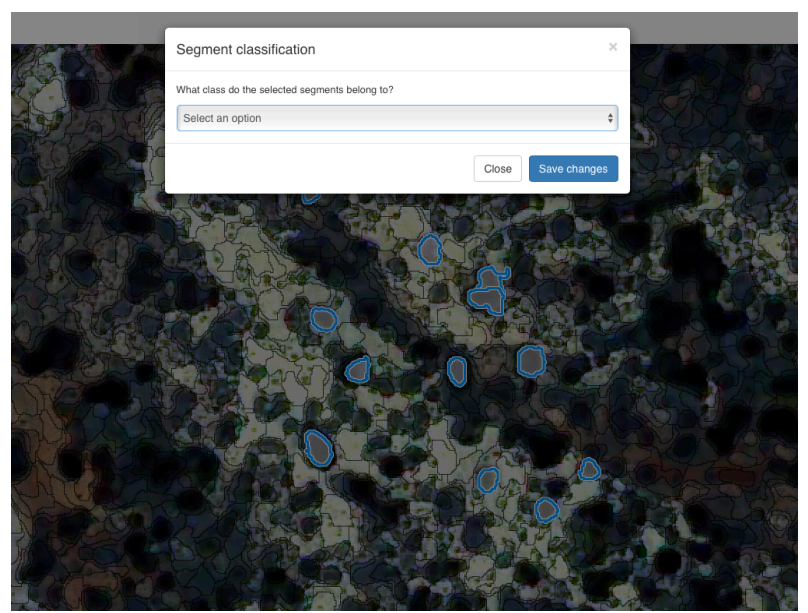

Figure 4. Manual labeling of several segments.

\section{EXPERIMENTAL RESULTS}

To prove the effectiveness of the proposed platform, a small experiment was carried out. A WorldView-2 (WV-2) dataset acquired on May 16th, 2011, was used for the analysis. The scene (show in Figure 3 is mostly characterized by scattered shrubs, bare soil, buildings and roads. Image annotation problem was reduced to the annotation of only two classes: non-shrub and shrub.

The use of the platform is described as follows: (1) An initial set of 50 regions was labeled by the user in order to start the AL approach. (2) The classifier was trained with the labeled elements. (3) Then 100 unlabeled segments were randomly selected by AL approach, the 5 elements most relevant were displayed to the user to label them manually. The segments are labeled one to one. To do this, the view is positioned in one of the elements to label, after this, the next element is displayed. (4) Steps 2 and 3 were repeated until reach a total of 50 iterations, which completed the training of the classifier.

A visual inspection after classifying 100 unlabeled regions points out a good correspondence between the obtained results (shown in Figure 5] and the expected by the user.

At this time, the platform is only available for the internal use of a small user group, however, it will open to a wider group in future releases.

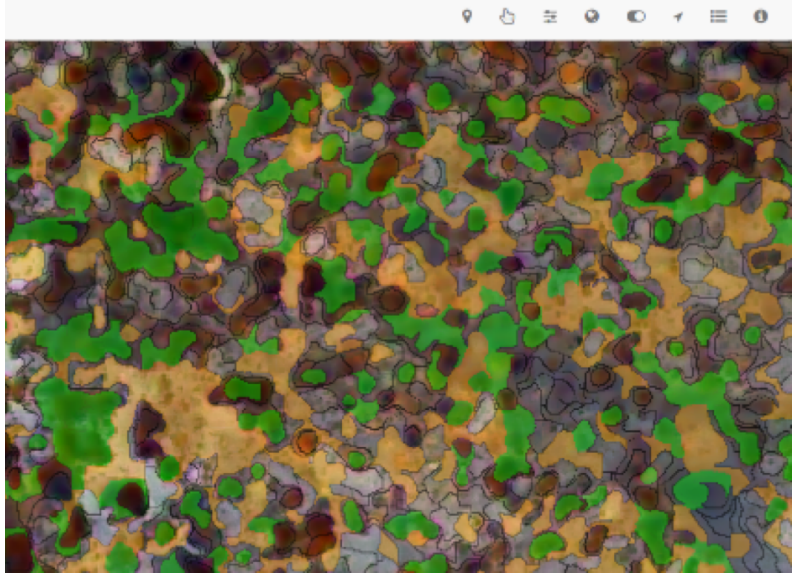

Figure 5. Results of the labeling process by the classifier trained by the AL approach.

\section{CONCLUSIONS}

A web-based platform for the annotation of remote sensing imagery was presented. The proposed platform allowed the interactive training, using an active learning approach, of a machine learning method for automatic image annotation. The use of the platform has facilitated the annotation of images without the need of large training set. The obtained results are promising, however some issues must be solved to improve the user experience. One of the main problems is the lack of an intuitive way to label more than one segment during the query made by the active learning approach. Another one is the lack of a feature extraction module. New releases of the platform will intended to solve these problems.

\section{ACKNOWLEDGEMENTS}

A. Garcia-Pedrero (grant 216146) acknowledges the support for the realization of his doctoral thesis to the Mexican National Council of Science and Technology (CONACyT). This work has been partially funded by FONDEF IT13I20002, and the Centro de Recursos Hídricos para la Agricultura y la Minería CRHIAM (CONICYT -FONDAP-1513001), and by the Universidad Politécnica de Madrid (AL16-PID-17).

\section{References}

Achanta, R., Shaji, A., Smith, K., Lucchi, A., Fua, P. and Susstrunk, S., 2012. SLIC Superpixels Compared to Stateof-the-Art Superpixel Methods. Pattern Analysis and Machine Intelligence, IEEE Transactions on 34(11), pp. 2274-2282.

Bengio, Y., Delalleau, O. and Le Roux, N., 2006. Label propagation and quadratic criterion. Semi-supervised learning.

Crawford, M. M., Tuia, D. and Yang, H. L., 2013. Active learning: Any value for classification of remotely sensed data? Proceedings of the IEEE 101(3), pp. 593-608.

Garcia-Pedrero, A., Gonzalo-Martin, C., Fonseca-Luengo, D. and Lillo-Saavedra, M., 2015. A geobia methodology for fragmented agricultural landscapes. Remote Sensing 7(1), pp. 767787.

Ma, Y., Wu, H., Wang, L., Huang, B., Ranjan, R., Zomaya, A. and Jie, W., 2015. Remote sensing big data computing: challenges and opportunities. Future Generation Computer Systems 51 , pp. 47-60. 
Olofsson, P., Foody, G. M., Herold, M., Stehman, S. V., Woodcock, C. E. and Wulder, M. A., 2014. Good practices for estimating area and assessing accuracy of land change. Remote Sensing of Environment 148, pp. 42-57.

Pedregosa, F., Varoquaux, G., Gramfort, A., Michel, V., Thirion, B., Grisel, O., Blondel, M., Prettenhofer, P., Weiss, R., Dubourg, V., Vanderplas, J., Passos, A., Cournapeau, D., Brucher, M., Perrot, M. and Duchesnay, E., 2011. Scikit-learn: Machine learning in Python. Journal of Machine Learning Research 12, pp. 2825-2830.

Stumpf, A., Lachiche, N., Malet, J.-P., Kerle, N. and Puissant, A., 2014. Active learning in the spatial domain for remote sensing image classification. IEEE Transactions on Geoscience and Remote Sensing 52(5), pp. 2492-2507.

Tuia, D. and Munoz-Mari, J., 2013. Learning user's confidence for active learning. IEEE Transactions on Geoscience and Remote Sensing 51(2), pp. 872-880.

Tuia, D., Ratle, F., Pacifici, F., Kanevski, M. F. and Emery, W. J., 2009. Active learning methods for remote sensing image classification. IEEE Transactions on Geoscience and Remote Sensing 47(7), pp. 2218-2232.

Xia, G.-S., Wang, Z., Xiong, C. and Zhang, L., 2015. Accurate annotation of remote sensing images via active spectral clustering with little expert knowledge. Remote Sensing 7(11), pp. $15014-15045$. 